\title{
GREGOR: Optics redesign and updates from 2018-2020
}

\author{
Lucia Kleint, Thomas Berkefeld, Miguel Esteves, Thomas Sonner, Reiner Volkmer, Karin Gerber, Felix Krämer, \\ Olivier Grassin, and Svetlana Berdyugina
}

\begin{abstract}
Leibniz-Institut für Sonnenphysik (KIS), Schöneckstrasse 6, 79104 Freiburg, Germany
e-mail: lucia.kleint@fhnw.ch
\end{abstract}

Received 20 April 2020 / Accepted 13 June 2020

\begin{abstract}
The GREGOR telescope was inaugurated in 2012. In 2018, we began a complete upgrade, involving optics, alignment, instrumentation, mechanical upgrades for vibration reduction, updated control systems, and building enhancements, and in addition, adapted management and policies. This paper describes all major updates performed during this time. Since 2012, all powered mirrors except for M1 were exchanged. Since March 2020, GREGOR observes with diffraction-limited performance and a new optics and instrument layout.
\end{abstract}

Key words. telescopes - Sun: general

\section{Introduction}

Solar telescopes have always striven to evolve in diameter and thus spatial resolution (e.g., review by Kleint \& Gandorfer 2017). Before 2000, their diameters remained smaller than $1 \mathrm{~m}$, with the exception of the Mc Math Pierce telescope at Kitt Peak, which mostly observed in the infrared, however (Penn 2014). A new generation of telescopes started in the twentyfirst century with the Swedish Solar Telescope (SST) in 2002. The SST has a clear aperture of $0.98 \mathrm{~m}$ and was optimized for a very high image quality. It routinely delivers impressive highresolution solar images, especially also at wavelengths in the blue (Scharmer et al. 2003, 2013). It was followed in 2009 by the Goode Solar Telescope (GST, Goode et al. 2010), which has a $1.6 \mathrm{~m}$ clear aperture. The GST, for example, has obtained flare images with the highest resolution to date (Jing et al. 2016).

GREGOR is Europe's largest solar telescope. It became operational a few years after the GST. Its $1.5 \mathrm{~m}$ diameter with an optical footprint of $1.44 \mathrm{~m}$ allows us to resolve structures on the Sun as small as $50 \mathrm{~km}$ at $400 \mathrm{~nm}$. The GREGOR project started with its proposal in 2000 (von der Lühe et al. 2001) and carried out a science-verification phase from 2012 to 2013. The state of GREGOR at that time was published in a series of articles in Astronomische Nachrichten Vol. 333, No. 9, in particular, the GREGOR overview by Schmidt et al. (2012). GREGOR was designed to explore solar features at smaller scales than other telescopes at that time. Its theoretical spatial resolution surpasses that of the SST and is similar to that of the GST, and all three telescopes have significantly improved their image quality with advanced adaptive optics (AO) systems (Schmidt et al. 2016; Berkefeld et al. 2018; Scharmer et al. 2019). Their designs differ, however; GREGOR focuses on high-precision polarimetry, which has enabled investigations of polarization signals as small as $10^{-4} I_{c}$ to detect spatial variations of turbulent magnetic fields (Bianda et al. 2018; Dhara et al. 2019). Another advantage of GREGOR is its potential for polarimetric night observations, which has been used to study the polarization of planets and thus their atmospheres (Gisler et al. 2016). A past drawback of
GREGOR was that its image quality did not reach the theoretical limit, partly because a risk was taken with untested technologies, such as silicon carbide mirrors, which could not be polished well enough, and partly because of design problems. These difficulties have recently been solved by replacing all silicon carbide mirrors with mirrors made of Zerodur, which can be polished to the required quality, and by redesigning the AO relay optics. GREGOR now operates at its diffraction limit. The goal of this paper is to summarize the recent upgrades and enhancements that were carried out from 2018 to 2020 . We only briefly summarize the general properties of GREGOR and we refer to the article series from 2012 for more details.

GREGOR obtained its name by being a Gregory system with three imaging mirrors (M1, M2, and M3) whose properties are summarized in Table 1. More than $99 \%$ of the sunlight is reflected away at the cooled primary field stop F1. Only a beam with circular diameter of $150^{\prime \prime}$ passes through its central hole to M2. The F1 field stop is recoated yearly, currently with an aluminum layer on top of a nickel layer. The mirrors M4-M11 are flat mirrors with the purpose of directing the beam into the optics laboratory one story below the telescope level. M8-M10 can be rotated about the optical axis, thus acting as a derotator, which compensates for the solar image rotation induced by the alt-az mount of the telescope. A schematic drawing of GREGOR is shown in Fig. 1.

\section{Optics}

\subsection{Redesign of the optics laboratory}

The original optics laboratory layout was devised during the GREGOR design phase before 2008. It focused on the firstlight instruments GRIS, a spectropolarimeter based on a grating spectrograph (Collados et al. 2012), GFPI, a dual-etalon spectroscopic imager (Puschmann et al. 2012), plus an associated broadband imager, and BBI as a stand-alone imager (von der Lühe et al. 2012). Guest instruments, such as ZIMPOL for high-precision spectropolarimetry (Gandorfer \& Povel 1997) 
Table 1. Mirror properties for M1-M3.

\begin{tabular}{lccc}
\hline \hline & M1 & M2 & M3 \\
\hline Focal length [mm] & 2506.35 & 519.40 & 1398.5 \\
Optical footprint for $150^{\prime \prime}[\mathrm{cm}]$ & 144 & 38.1 & 27.5 \\
f\# & 1.7 & 1.4 & 5.1 \\
Curvature radius [mm] & 5012.70 & 1039.79 & 2797.00 \\
Conic constant & -1 (parabolic) & -0.306 (elliptic) & -0.538 (elliptic) \\
\hline
\end{tabular}

or the GREGOR planet polarimeter (GPP, Gisler et al. 2016), were also regularly operated. The AO was mounted on a vertical bench, which saves space, but may be disadvantageous in terms of vibrations and alignment. The beam is collimated for the AO after F3 via M12 and then reimaged via M15. The AO itself consists of a tip-tilt (M13) and a deformable mirror (DM, M14). The setup is shown in the top panel of Fig. 2. During the past two years, we realized that there were difficulties with beam stability, alignment, and the optical quality induced by the two biconic mirrors M12 and M15. These mirrors originally featured biconic shapes to minimize the astigmatism that arises due to an oblique incidence on a focusing mirror. However, we realized that they created field-dependent aberrations (mostly astigmatism and coma) that are prominent at the design angles. Unfortunately, M12 and M15 could not be aligned arbitrarily to minimize the aberrations, which never fully vanished, however, because of a fixed focal plane and vignetting at other optical elements. Their alignment tolerances were also too strict. Additionally, there was a lack of space in the optics laboratory to develop and install new instrumentation. Therefore we completely redesigned the optics after M11, including new offaxis parabolic mirrors to replace M12 and M15 and an improved instrument layout.

The new layout was devised based on the following criteria:

- There shall be more space for the science instruments and a future multi-conjugate adaptive optics (MCAO) via a different beam distribution while keeping all current capabilities.

- The setup of the AO relay optics shall be horizontal (for vibration reduction, stability, and easier alignment).

- The (total) angle at M12/M15 shall be $=8^{\circ}$ (the two mirrors compensate for each other). Angles $<8^{\circ}$ are not possible because of the BS plate between M15 and M16.

- There shall be 1:1 imaging of the AO relay optics between $\mathrm{F} 3$ and F4. The exit pupil shall be telecentric (at infinity).

- The image quality in F4 shall be perfect over a radius of $60^{\prime \prime}$ (=2 arcmin image diameter).

- F3 shall be located at least $1000 \mathrm{~mm}$ after M11.

- The pupil size is limited by the DM size.

- A visible/infrared (VIS/IR) BS between M15 and M16 reflects the VIS, only IR passes through toward GRIS. This improves the antireflection coatings for the VIS and the IR elements. This BS is mounted on a rotating table and can be exchanged for instance by a 50/50 BS for special setups that require other wavelength distributions.

- The angles at mirrors shall be small for polarimetry.

- DM-M15 shall be parallel to M11-M12 (this is not strictly required, but it simplifies the alignment).

- F4 IR shall remain unchanged (1420 mm from M11). M16F4 IR is on the same line as M11-F4 IR to maintain the beam angles, such that the GRIS instrument does not require any changes.

- The wavefront sensor (WFS) shall be located after all powered mirrors.

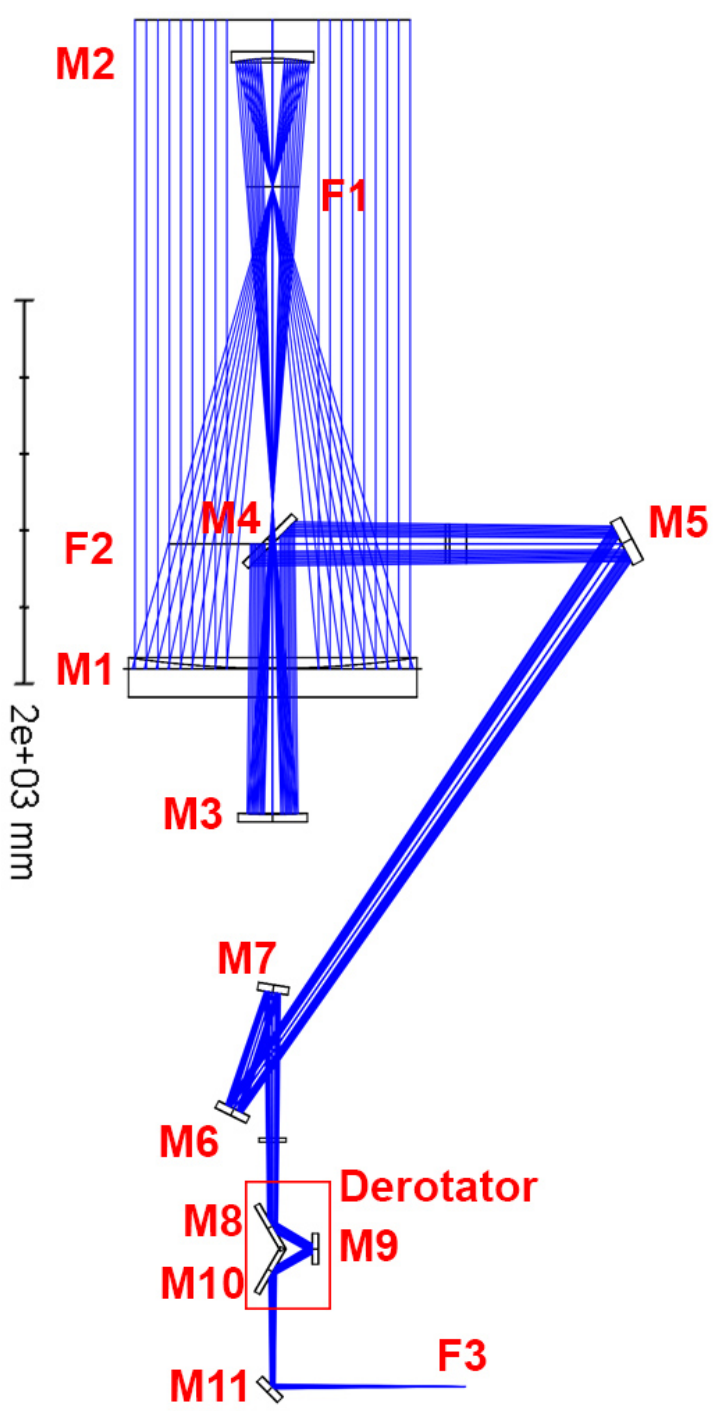

Fig. 1. GREGOR optical layout until F3.

The original and the new design are shown in Fig. 2. The new off-axis parabolic (OAP) mirrors M12 and M15 are identical and compensate for each other's aberrations. Their paraxial focal length is $1978 \mathrm{~mm}$ and the off-axis distance is $277 \mathrm{~mm}$. They are made from Zerodur with a diameter of $125 \mathrm{~mm}$, thickness of $20 \mathrm{~mm}$, with a silver coating. Their measured surface error RMS is 6.9 and $5.2 \mathrm{~nm}$ for the two mirrors, and this quality was achieved with two ion beam figuring (IBF) polishing runs per mirror.

The original and new MTFs and spot diagrams are shown in Fig. 3. The MTF for different field angles improves with increasing wavelength, but the spatial resolution obviously decreases at higher wavelength. We therefore show the MTF at $500 \mathrm{~nm}$ to illustrate the problems with loss of contrast of the original setup and the much better performance of OAPs. All plots assume a maximum field of view (FOV) of 120".

Figure 4 shows the new instrument layout and a photo of the setup in March 2020. GRIS and the slitjaw system remain. The new Fabry-Perot Interferometer (FPI), currently under development, will be in the straight beam and has very little reflections. Parallel to it is its broadband channel, for which we plan phase diversity, and a fast imager in the blue. An insertable mirror, which can be rotated, sends the light either to a visitor table or to 

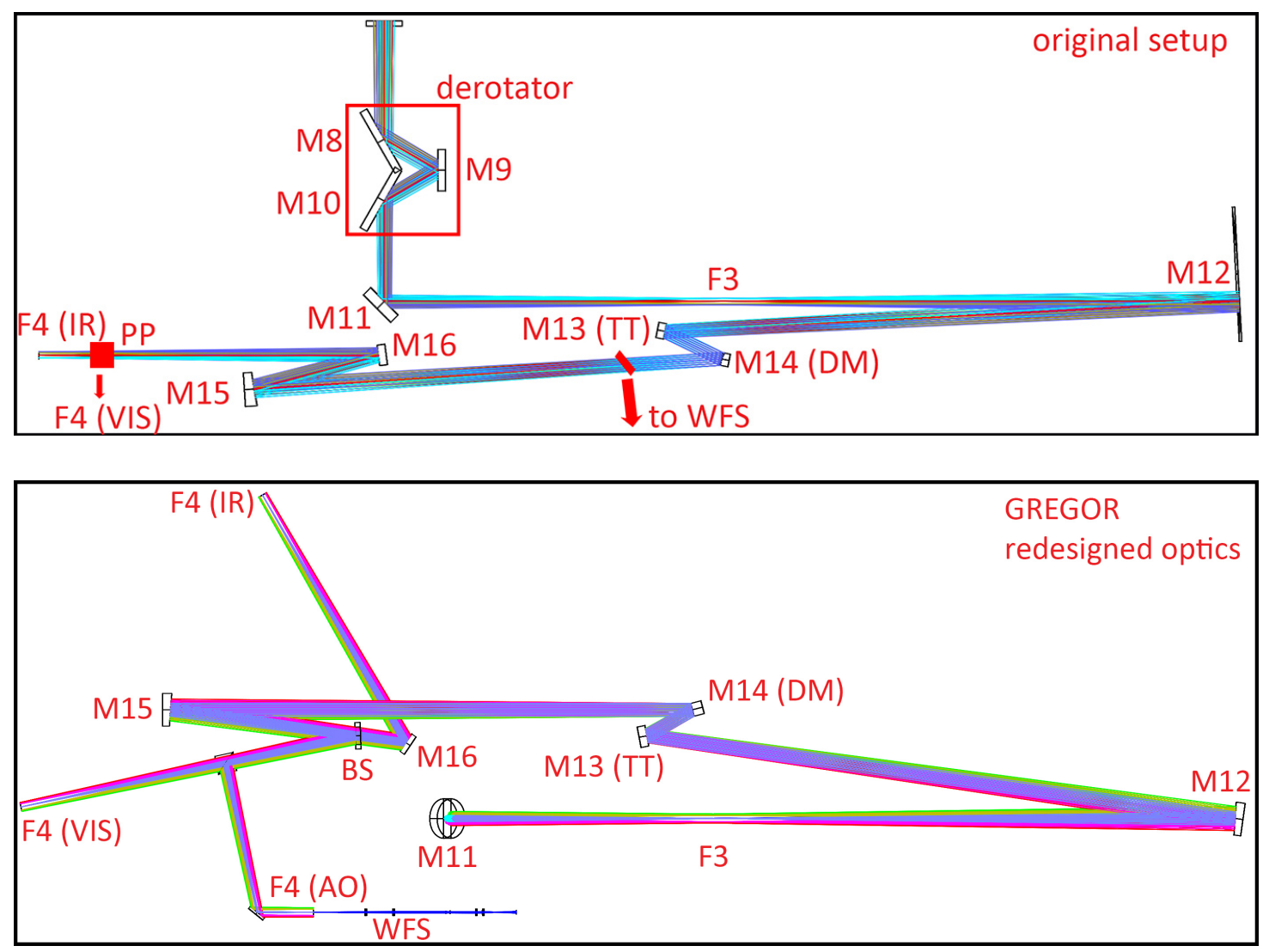

Fig. 2. Top image: original GREGOR setup in side view from the vacuum exit window to F4. The location of the wavefront sensor (WFS) is indicated with an arrow. The wavelength splitting was made with a pentaprism (PP), and the instrument focal planes (F4) are shown for IR and VIS. Bottom image: new setup in top view. The VIS beam is reflected on the front side of the beamsplitter plate (BS). It is then split by a beamsplitter with a fraction of the light going into the AO. The IR beam passes the BS and is then reflected on M16. The WFS is located after all mirrors with power.

GFPI/HIFI, which is now located near the entrance to the optics laboratory. One table is saved for a future upstairs spectrograph, which can be fed by rotating M16. We were able to use about $75 \%$ of the mechanical parts and adapted them for the new setup, which accelerated the construction and fabrication.

The new design has many advantages: By having a constant beam height above the optical tables, all alignment is simplified. The off-axis parabolas have much better alignment tolerances than the former biconic mirrors. The point spread function (PSF) remains diffraction-limited in all field points, even for small deviations of properties (e.g., conic constant difference of $2 \%$, decenter of $5 \mathrm{~mm}$, or $1 \%$ deviation in paraxial radius). The MTF remains nearly constant across the whole FOV, compared to a $\sim 50 \%$ drop in the original setup. There is space for a future MCAO and a future upstairs spectrograph, while all current instrument capabilities remain. Additionally, all optical elements are accessible. Furthermore, all critical optical elements are motorized, so that their alignment is reproducible. During the redesign, the number of motorized elements was increased from 7 to 13 , and the motorization control interfaces were updated for manual and remote control. The wavefront sensor is located after M15 and thus is able to correct for any static aberrations induced by the relay optics. The wavelength splitting is performed using a dichroic BS plate and no longer a pentaprism, which is an advantage for polarimetry (smaller angles). By using different BS plates, it is possible to change the wavelength cutoff for the visible beam, currently either to $650 \mathrm{~nm}$ or $900 \mathrm{~nm}$. The location of the BS plate in the beam simplifies the IR and visible coatings because now only the rear side of the BS plate requires a broad antireflection coating, while instruments receive limited wavelength ranges and therefore can use mostly standard antireflection coatings, which are easier to obtain.

The new optical setup was installed in March 2020. The optical elements were aligned with a laser, which traced the beam backward from F4. The laser direction was aligned in azimuth from the original F4 GRIS location to a plummet hanging from the exit window, which defined the vertical optical axis of the telescope. During the backward-tracing, the beam angles were measured for M15, the DM, and M12, and the mirrors were shifted and tilted as necessary to match the design values. The laser was reflected upward into the vertical optical axis by M11, where it coincided with the plummet line. The wavefront sensor was only adapted to its change of location, but its properties remain. We then calibrated the AO by applying Hadamard shapes to the DM and measuring the response of the wavefront sensor. This gave us the response of the 256 actuators. A pinhole in the AO F4 was used to measure the subaperture reference positions for the perfect wavefront. Then we locked the AO on a USAF target in F3, which was immediately sharp over the whole FOV in the science camera in F4 VIS. The improvement compared to the old relay optics was immediately noticeable and can be attributed to the pair of off-axis parabolic mirrors with a small and equal reflection angle.

Figure 5 shows a test target recorded on March 21, 2020. It was recorded directly in the visible F4, with an exposure time of $3 \mathrm{~ms}$ at $393.55 \pm 1.1 \mathrm{~nm}$. No AO mode offsets were applied or 

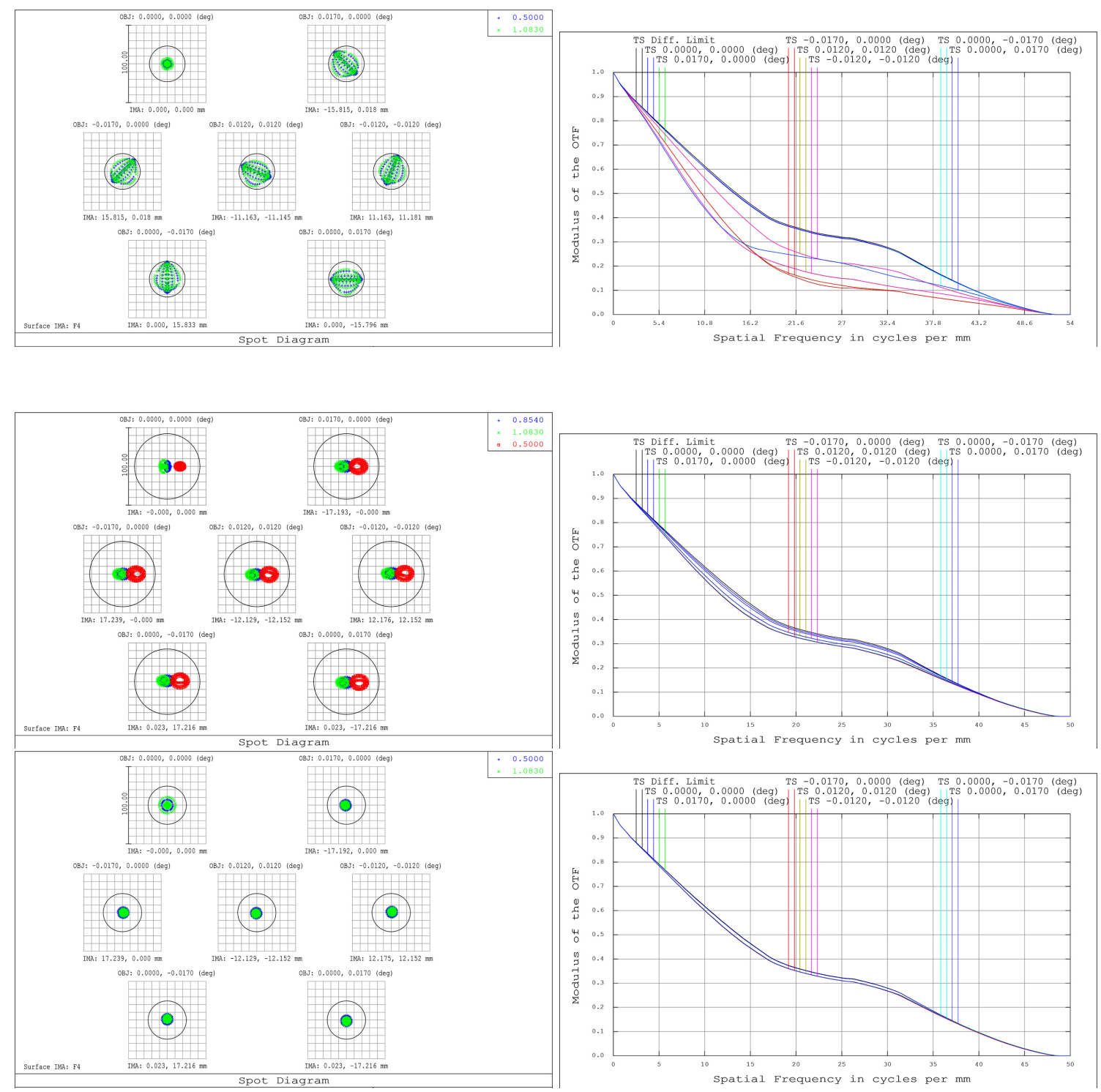

Fig. 3. GREGOR spot diagrams for different wavelengths and MTF for $500 \mathrm{~nm}$ with max. FOV $=120^{\prime \prime}$. The circle indicates the Airy disk. Top row: original M12 and M15. Diffraction-limited performance is only achieved for the center of the FOV with a very fast drop in contrast off-center. This simulation was made for ideal M12 and M15 mirrors, and we suspect that their specifications were not met, which would further worsen their properties. Middle row: new IR setup. The lateral shift is due to the BS plate, and $500 \mathrm{~nm}$ (red) is displayed to show that even the ZIMPOL guest instrument setup, which uses VIS in the IR beam by using a custom beamsplitter plate, would still have a diffraction-limited performance. Bottom row: new visible setup with basically no loss of contrast for the whole field of $120^{\prime \prime}$. All MTFs have good properties in the IR, and therefore $500 \mathrm{~nm}$ is shown here.

necessary. The pixel scale is $00^{\prime \prime} 023$ pixel $^{-1}$. The figure is a composite of two images: the camera was shifted in height to record all subtargets. This figure demonstrates that the redesign was successful because all targets are sharp across the whole FOV and the correct element (fifth group, fifth element) is resolved in all subtargets, some of which are shown magnified in the blue boxes. No astigmatism or coma is visible. For comparison, the orange boxes show a test target, taken with the original optics at $396 \mathrm{~nm}$ on July 19, 2019. It is clearly visible that previously, locations outside of the image center showed strong astigmatism ("shadows"), consistent with the MTF shown in Fig. 3.

\section{2. $M 2$}

The original secondary mirror (M2) was fabricated from silicone carbide (Cesic), which is a material of high stiffness whose thermal properties are favorable to absorb the $1.3 \mathrm{~W}$ from the incoming sunlight after the primary field stop when we assume that each mirror absorbs $10 \%$ of its incoming light. Unfortunately, the Cesic surface could not be polished well enough, and at the end of the polishing the original M2 had an rms wavefront error (WFE) of $\sim 40 \mathrm{~nm}$, mostly in the shape of concentric rings, as shown in Fig. 6. These rings were of the same scale size as the AO subapertures, which means that the $\mathrm{AO}$ was unable to "see" them and therefore was not able to correct for them. These mid-frequency errors lead to a severe reduction in contrast both in the WFS and in the science camera. The low WFS contrast then leads to a low signal-to-noise ratio of the wavefront reconstruction and a reduced performance of the $\mathrm{AO}$, especially when locking on granulation. Furthermore, the unsensed midfrequency errors lead to spatial aliasing, which means that insufficient spatial points are available to determine the wavefront, 

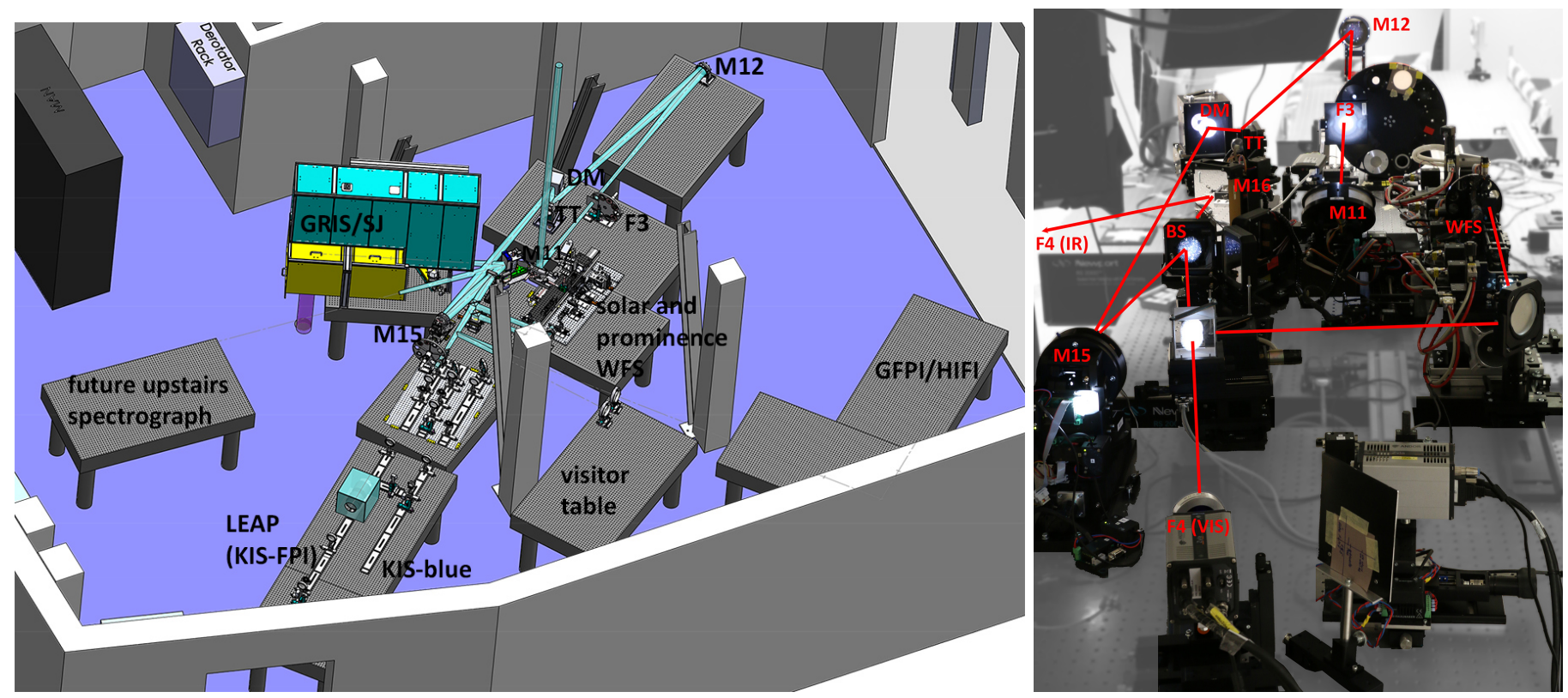

Fig. 4. Left: new instrument layout at GREGOR. GRIS (yellow) with the SJ system (green box above GRIS) are the only optics that remain in the same place, but all original instrument combinations remain. The AO is now horizontal and the new KIS FPI is drawn toward the window. The empty tables are for temporary visitor setups and for GFPI/HIFI. Right: photo of the setup as installed in March 2020. The red line traces the beam.

and they result in non-optimal wavefront reconstruction and correction or in an increased wavefront error, which is why we were unable to fully exploit the maximum number of corrected modes in the past.

It was therefore decided in 2014 to replace M2 with a mirror made of Zerodur. A call for tender was issued, this time with a much stricter specification on the allowed WFE. The WFE $\Phi$ was specified in terms of a structure function $D_{\Phi}(r)$, which describes the error at different spatial scales:

$D_{\Phi}(r)=\operatorname{avg}\left([\Phi(x+r)-\Phi(r)]^{2}\right)$,

where $r$ denotes different separations of (randomly) selected points. The outer diameter was specified to $430 \mathrm{~mm}$. Because M1 is stopped down to $1.44 \mathrm{~m}$ due to its polishing quality, the optical footprint on M2 is $381 \mathrm{~mm}$, but up to $385 \mathrm{~mm}$ are of sufficient polishing quality.

There were some difficulties during fabrication and the specifications could not be met with conventional polishing methods. However, after using IBF, the mirror surface is now one of the best at GREGOR. The new M2 was installed in July 2018, and the improvements in contrast after its installation at GREGOR were noticeable even before any acceptance testing was carried out. They are difficult to quantify, but we estimate an improvement in the contrast of a factor 1.5-2 in the WFS. Since then, the adaptive optics easily locks on granulation, even during bad seeing. Figure 6 shows the interferograms of the original and the new M2. To display both on the same scale ( $\pm 80 \mathrm{~nm}$ WFE), data from the original M2 had to be clipped. Figure 7 shows the structure functions of original and new M2 and the specifications from the call for tender, which were clearly exceeded. This was the last Cesic mirror to be replaced, and GREGOR now contains only Zerodur mirrors.

\subsection{Derotator}

The derotator consists of M8-M10 and was installed in 2016. M8 and M10 are tilted by $30^{\circ}$, leading to incident beam angles of $60^{\circ}$, which is not optimal for polarimetry and requires careful calibration (Hofmann et al. 2012). By rotating about the optical axis, the derotator counteracts the image rotation introduced by the alt-az telescope mount. The image rotation is the sum of parallactic angle + azimuth - zenith distance - solar p0 angle + an offset for the desired orientation. The required derotator rotation is half of the image rotation. The image rotation rate is highest in June when the Sun nearly reaches zenith and exceeds $5^{\circ}$ per minute then, while it generally remains below $0.5^{\circ}$ per min before 11 UT during nearly the whole year.

The original developer GUI was used by observers to control the derotator for several years. In 2019 we enhanced the derotator control in functionality and design. The derotator can now be controlled from the GREGOR GUI, and we calibrated its orientation, such that observers can simply insert the desired solar angle (e.g., 0 for north-south) for the spectrograph slit orientation and all calculations are performed internally.

\subsection{Alignment}

GREGOR has very tight alignment tolerances because of its fast beam. It is therefore nearly impossible to align the telescope visually (using lasers), and past experience has shown larger aberrations after each recoating. It is possible that each M1 removal and remounting has modified its position because even a 0.001 -degree tilt is noticeable in the aberrations measured by the AO, but not visually. In the past, M2 was shifted and tilted to counteract these aberrations, but after the fourth recoating in 2017, the required shifts reached several millimeter.

This problem was solved in two ways: (1) We devised a new method to align M1 by using the AO and successfully carried out the alignment in May 2019. The method involves using the force actuators on the back of M1 and creating a response matrix of each actuator. It can then be calculated in which positions the actuators should be to minimize the aberrations. The finetuning is made afterward by direct feedback from the AO. We found that the actuators for tilt had to be moved significantly in 


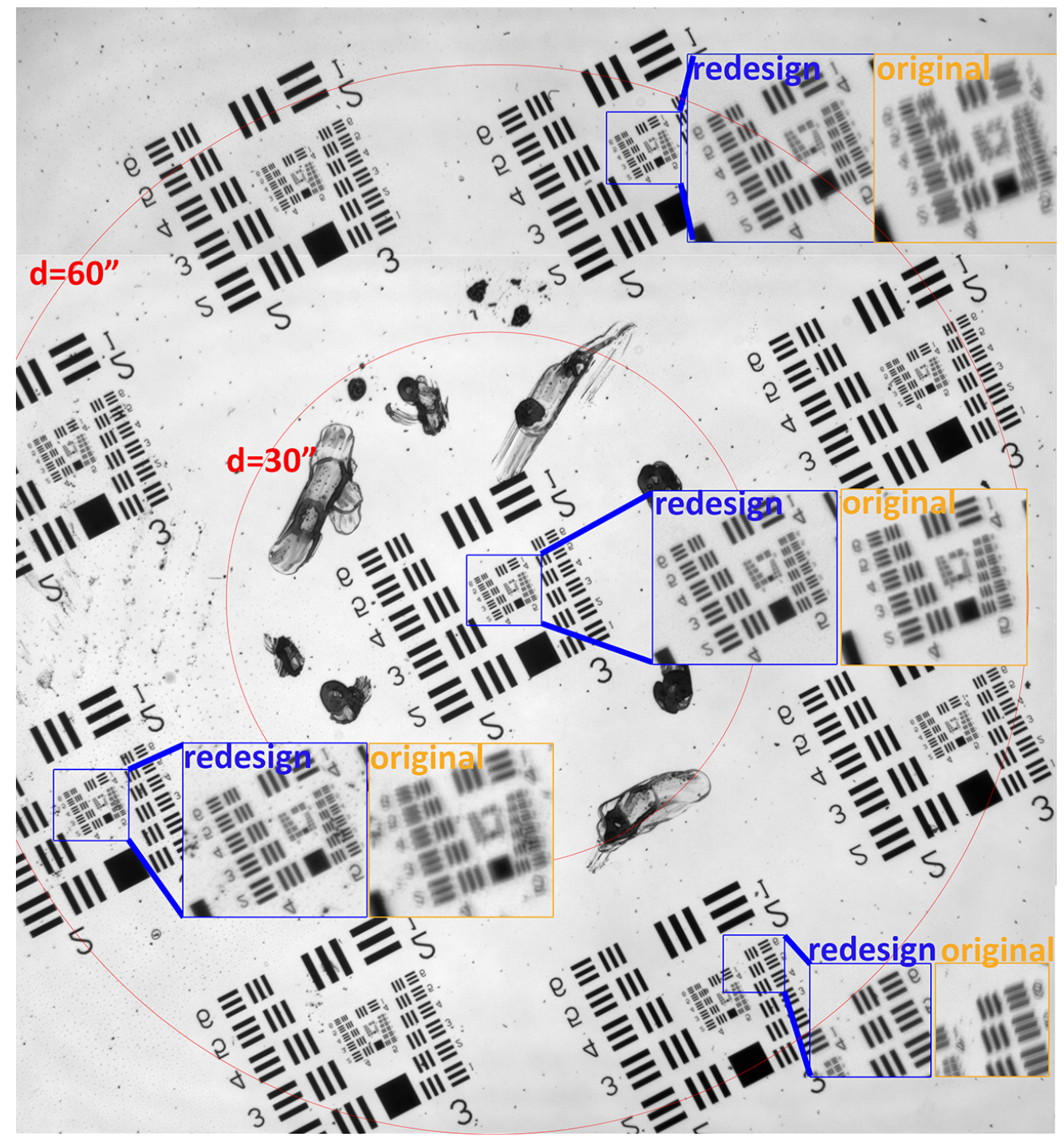

Fig. 5. Image of a test target obtained after the redesign. The blue insets show magnified sections, and the orange insets demonstrate the comparison to the original optics setup, which suffered from astigmatism and coma outside of the image center. The redesigned optics are perfectly sharp across the whole field of view. The image scale is indicated with red circles, whose diameters are specified in the figure.

May 2019, which may support the hypothesis that remounting M1 introduces mirror tilt. In the future, we will explore making M1 actively controlled, which would enable us to perform this procedure in minutes instead of days.

(2) Because M1 and M2 are not perfectly aligned with the optical axis, even by the above procedure, a beam wobble is introduced downstream. For the remaining small beam deviations, we programmed a beam tracker, which consists of a webcam pointing to $\mathrm{F} 3$ and a program that automatically monitors the beam position and sends signals to tilt M5 to compensate for the diurnal variation. The beam-tracker program fits a circle to the beam in F3 and keeps its position constant, which stabilized all focal planes after F3. Full stability can only be achieved when the pupil is also stabilized. This is done by measuring the intensity distribution on the WFS and an offset of the pupil is therefore directly visible. The AO controls the tilt of M11, and because the DM is exactly in a pupil plane, all subsequent pupils are stabilized. M11 is located $1000 \mathrm{~mm}$ from F3, while M5 is located $808 \mathrm{~mm}$ from a pupil. While both mirrors are not perfectly located in pupil or image planes and therefore affect the image and pupil motions, the effect on the pupil (when M5 is moved) or the image (when M11 is moved) planes is very weak, and both trackers converge iteratively within seconds to a centered image in F3 and pupil on the DM.

\section{Infrastructure}

\subsection{Vibrations}

Vibrations are a common problem in telescopes, and depending on their frequencies, they cause significant problems in the data. We measured vibrations at GREGOR and removed several culprits that introduced vibrations: the mirror coolers were insulated better from the floor, an optical table was replaced with a more stable version, the AO setup was fortified with additional struts before it was made completely horizontal, additional supports were constructed for the slitjaw system, and the air conditioning is being investigated because we observed it to cause vibrations at 50,60 , and $125 \mathrm{~Hz}$. We found that the air used for the cooling of M1, which is distributed to the backside of M1 by fans, apparently causes vibrations of M1 of about 200 and $390 \mathrm{~Hz}$. Vibrations below $100 \mathrm{~Hz}$ can be mostly corrected for by the AO system, but the peaks around 200 and $390 \mathrm{~Hz}$ unfortunately cannot and are also seen in AO power spectra. Their 

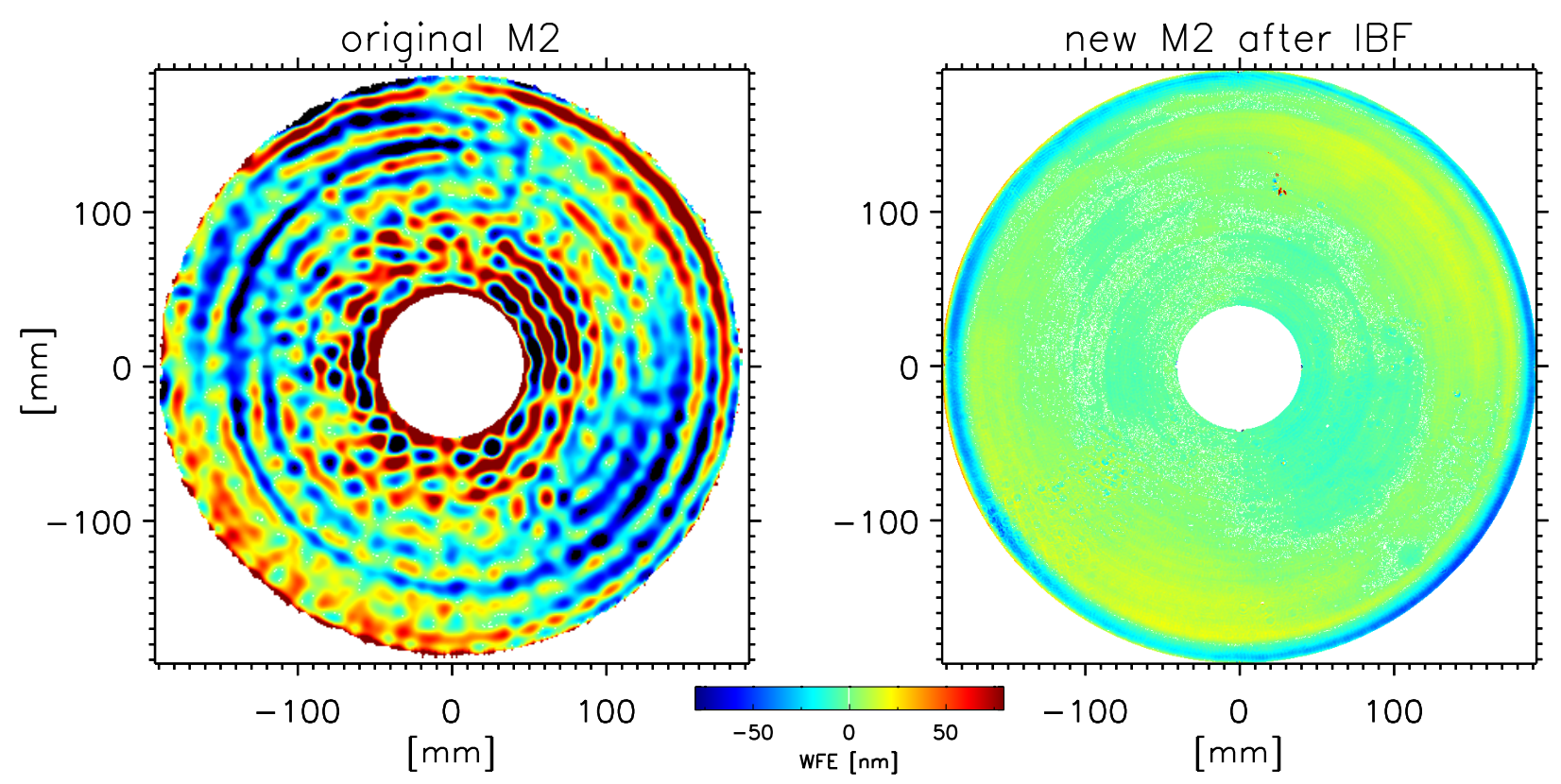

Fig. 6. Comparison of the interferograms of original and new M2. The display range is $\pm 80 \mathrm{~nm}$ for both, showing wavefront errors, which are a factor of 2 larger than the surface errors. Some dust and fingerprints explain the small imperfections on the interferogram of the new M2. The WFE rms is $39 \mathrm{~nm}$ and $8 \mathrm{~nm}$ for the original and new M2, respectively.

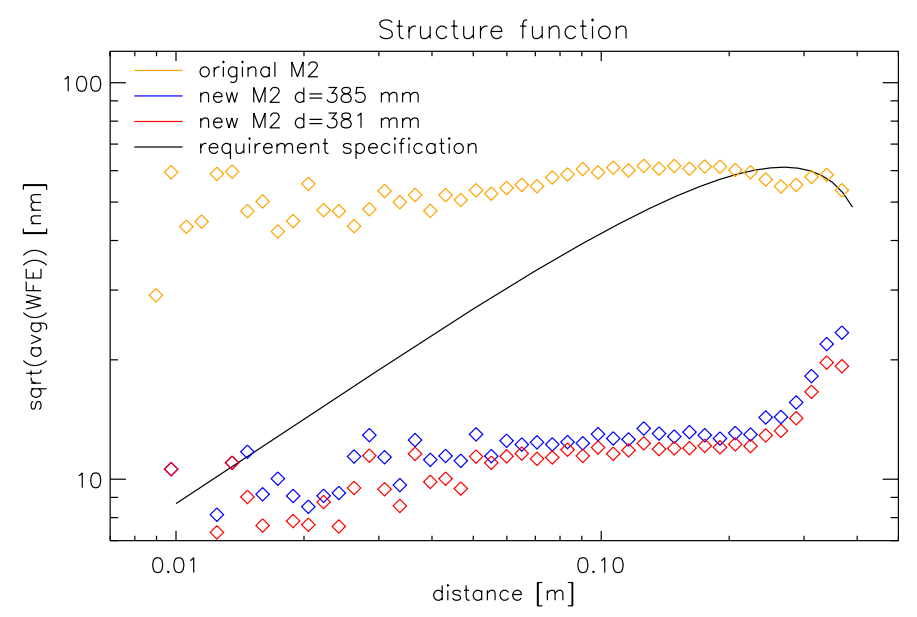

Fig. 7. Structure function. $10^{6}$ randomly selected points were used to calculate differences in the WFE according to Eq. (1). The new M2 is significantly better than the requirements. The larger scatter near smaller spatial scales is due to averaging fewer points when binning. The current optical footprint on M2 is $381 \mathrm{~mm}$.

amplitude can be reduced by reducing the fan speed of the M1 cooling, and in certain outside temperature conditions, it is possible to reduce the amplitude by $\sim 75 \%$. However, a universally applicable solution is currently being investigated because a first attempt of stiffening the housing of the coolers did not reduce the vibrations. In general, the combined measures taken so far have reduced the overall vibrations by at least a factor of 10 .

\subsection{Seeing}

As the air surrounding the telescope heats up during the day, the image quality degrades ("seeing"). At GREGOR, this often translates into $r_{0}$ values that drop by a factor of 4 from early morning to afternoon. We therefore repainted all panels on the east side of the building with a special paint that keeps the panels within one degree of the ambient temperature by emitting in the infrared. Additionally, we repainted the flagstones and the tiles on the roof of the building with special paint based on titanium oxide. Our temperature measurements with an infrared camera have shown that the floor temperature decreased by at least 2 degrees.

Temperature logging in the optics laboratory, which was installed in April 2019, shows diurnal variations with an amplitude of about $0.5^{\circ} \mathrm{C}$, which may contribute to the seeing in the optics laboratory; their cause is being investigated. As a first step, we created a new server room adjacent to the optics laboratory and are in the process of relocating most electronics and computers that contribute to heating the optics laboratory.

\subsection{Electric installations}

In 2017, the old uninterruptible power supply (UPS) was replaced with a new three-phased UPS, which doubled the power output (to $40 \mathrm{kVA}$ ). The new control of the UPS was programmed by KIS and installed by a company. Since February 2019, all critical systems of GREGOR are on UPS. During power outages, the UPS bridges the time until the Diesel generator starts. In case of catastrophic failures, the UPS has enough power to supply GREGOR for about $30 \mathrm{~min}$, which allows for a controlled shutdown, especially of the dome, which must be closed to protect the telescope from weather.

\subsection{Documentation}

Changes that are not documented can lead to frustration when systems behave differently than at the last time. We therefore introduced a version-controlled repository of GREGOR documents, partially open to the public ${ }^{1}$. These include manuals, checklists, technical notes, and requirements specifications. All

\footnotetext{
1 http://www.leibniz-kis.de/de/observatorien/gregor/ documentation/
} 
instruments now have checklists that are also prominently displayed in the observing room, which simplifies the observations and leads to fewer missing calibration data, for example. Additionally, every observer writes an observing report after their observing run to detail problems (if any), and every maintenance is documented with maintenance reports that are accessible to all partners on an internal website. Errors and bugs are reported through a ticketing system for better traceability of all actions.

\subsection{Observing room}

The GREGOR observing room is located on the third floor, two floors below the optics laboratory. It features three control computers, one of which is usually used for telescope and AO controls, and the other two are available to control instruments. The screens can be mirrored in Freiburg for remote observations or observing support. We installed four webcams at the top of the neighboring VTT telescope to monitor the clouds, and a new weather screen is mounted in the observing room showing the $360^{\circ}$ view, solar activity, and seeing and weather plots. The background automatically turns red when a weather limit is exceeded, for example, if the wind exceeds $20 \mathrm{~m} / \mathrm{s}$ or if the humidity exceeds $80 \%$, indicating that the telescope has to be closed. This has solved discussions between observers and assistants whether the telescope should or should not be closed.

\section{Instrumentation}

In this section we only review upgrades made by KIS from 2017 to 2020. There were no changes to BBI, the GREGOR planet polarimeter (GPP), and the guest instrument ZIMPOL.

\subsection{Slitjaw system}

The slitjaw (SJ) system is a context imager for the GRIS instrument. It was completely redesigned in 2018 to provide diffraction-limited images. The GRIS slit mask and integral field unit (IFU) mask are inclined by $15^{\circ}$, which reflects the beam upward (beam angle $2 \times 15^{\circ}$ ), as shown in the optical layout in Fig. 8. A motorized lens with a focal length of $750 \mathrm{~mm}$ automatically adjusts its position depending on the slit position during a scan to keep a constant distance between the (inclined) slit mask and the lens. The light is then distributed onto three horizontal rails above the SJ table. The lowest rail can be used for temporary setups. The middle rail receives $80 \%$ of the incoming light and features an $\mathrm{H}-\alpha$ Lyot filter with a passband of $0.4 \AA$ for chromospheric context images. The upper rail contains a $777 \mathrm{~nm}$ filter and shows photospheric context images. Both context images use a $f=300 \mathrm{~mm}$ lens, which together with Prosilica GT2050 cameras with $2048 \times 2048$ pixels $(5.5 \mu \mathrm{m} \times 5.5 \mu \mathrm{m})$ lead to a plate scale of 0 ’.05 pixel $^{-1}$.

For stability reasons, the whole SJ/GRIS optical table was replaced, which reduced vibrations by one order of magnitude. Additionally, supports were constructed that stabilize the overhanging part of the setup. A slit-scanning device was developed by building the mechanics and by programming the controls, which allows us to scan areas of the Sun.

\subsection{Adaptive optics}

The numerous enhancements of the GREGOR AO (Berkefeld et al. 2012) will be described in a forthcoming publication, and only a short summary is given here. The main

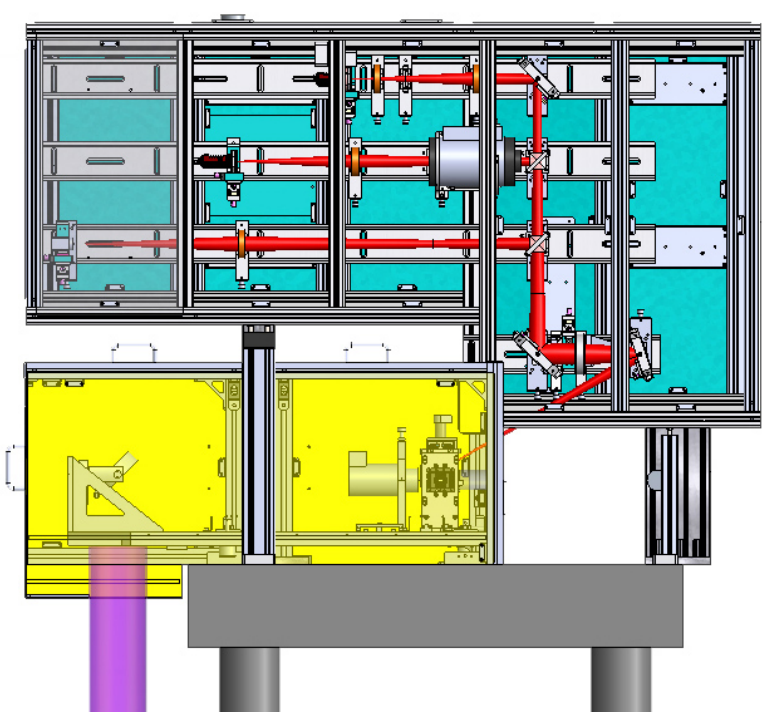

Fig. 8. Slitjaw system installed at GREGOR. The upper two channels are continuum and $\mathrm{H}$-alpha, respectively, while the bottom channel is currently not used.

enhancements of the AO include that the AO takes control of the telescope in closed-loop operations. By sending small positioning commands to the telescope, based on the tip-tilt measurements, the AO can track the desired feature, thus compensating for solar rotation, or positioning errors that may exceed the range of regular tip-tilt correction. It also controls the $\mathrm{z}$-position of $\mathrm{M} 2$ for the optimal focus, which needs to be adapted during the day because temperature and gravity vector changes lead to small variations in the focal distance between M1 and M2, but which are too large to be corrected for by a simple focus offset via the DM. Furthermore, the AO automatically sets the ideal number of modes for the correction, depending on the seeing and the target, and can also update the reference image periodically if desired.

\section{Control systems}

Most of the GREGOR GUIs were designed to be engineering GUIs and are therefore not particularly user-friendly for firsttime observers. Before 2019, an observer therefore had to use more than ten separate GUIs to control the telescope functions. A workshop was organized at KIS with representatives from each institute (KIS, MPS, AIP, and IAC) during which requirements for a new observing GUI were defined that would simplify the operation and display all relevant functions, and also add some new functionality, in one GUI. The new GUI was developed within a year, and it is operational since 2019.

It is programmed in QT and based on a publisher-subscriber principle using MQTT as protocol. A data-aggregation service collects all data from all subsystems and provides these data, together with external data such as current solar images published by other dedicated services, via topics on an MQTT broker. Multiple subscribers, for instance, GREGOR GUIs, can subscribe to these topics to be updated if their content changes. The advantage of this approach is that future applications, for example, a weather status page, can also be programmed to subscribe to the MQTT broker to obtain the relevant data. This will avoid previous problems when too many simultaneous commands created trouble with the network and system load. Safety-relevant features, such as who can take control, had to be considered in 


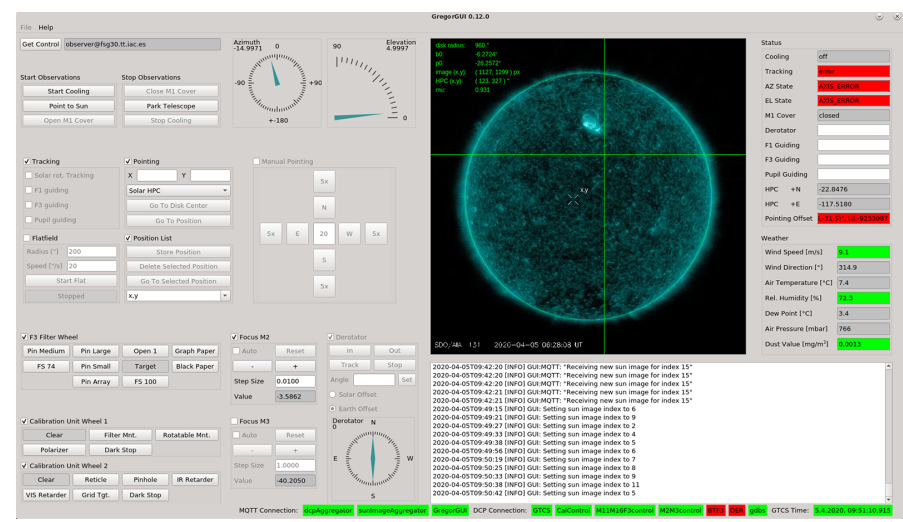

Fig. 9. GREGOR GUI. It allows us to control all essential telescope systems, such as positioning, pointing, filter wheels, calibration optics, focusing, and the derotator. The right side contains a display of current solar data, a system and weather status, and an event log.

addition to user-friendliness and simplicity of operation: User IDs are published using the same underlying mechanism by creating a UID-specific command topic, and the commander service subscribes to this to receive commands from only one commanding instance (e.g., a GREGOR GUI instance) at a time. The layout of the GUI is shown in Fig. 9 and was structured such that telescope operation is at the top, then pointing, positioning, and flatfielding, and control of motors (filter wheels, derotator, and focusing) at the bottom. The right side of the GUI displays the solar image in any desired SDO wavelength, status data (weather, systems, and seeing), and a log file.

\section{Management}

\subsection{Two observing seasons}

In addition to the optical and facility upgrades, we also introduced policy changes. One was to make the observing-time planning more flexible by having two observing seasons each year. Season 1 normally lasts from April to August and season 2 from August to December. This greatly facilitated dealing with unexpected difficulties, such as mirror recoatings that became urgent during the year. It also is advantageous for scientists when they do not have to wait for a year to apply for observing time if they have a good scientific idea immediately after a deadline.

\subsection{Access and permissions}

The other policy change was a much stricter control of telescope access and the limitation of personnel that is allowed to modify optics. Visitors no longer suddenly appear in the optics laboratory during observations. The alignment is also much more stable, and there are fewer unexpected changes and problems. After two years of observing with the new system, the observing reports are generally very positive, and campaigns without good data are rare.

\subsection{Technical maintenance and assistance}

GREGOR is a very complex telescope for its size. It is operated with a technical observing assistant on each observing day. The assistant is responsible for the telescope operation and the hardware. About 200 person-days are spent for assistance, with assistant campaigns lasting two to three weeks. While this number is high, having qualified operators for the telescope and AO increased the productivity of GREGOR significantly.

Technical maintenance is performed mostly during the winter break. More than 500 person-days are spent annually on technical work at the telescope, ranging from recoating mirrors, optics, mechanics, electronics, to updates of the infrastructure.

\section{Effect of the upgrades on observations}

Observers at GREGOR will notice many changes and enhancements. It is now possible to keep the AO locked on the quiet Sun for time periods exceeding a few dozen minutes. For objects with high contrast, such as sunspots and pores, the potential observing time spans hours. Observers will also notice a strong increase in image sharpness, especially outside of the central area of the FOV. This should enable studies of larger regions, for example, active regions, including penumbrae, flows, large filaments, or flares. In terms of user-friendliness, the enhancements of the derotator, the control systems, especially the GREGOR GUI, and the technical infrastructure, allow observers to quickly point the telescope to the desired position and image orientation, with fast changes possible for target-of-opportunity observations. Observers can also expect to be able to work more efficiently because documentation and checklists are available for instruments and systems, which avoids missing important calibration data, for example.

Several further enhancements are planned for future observations: A new spectropolarimeter based on Fabry Perot interferometers is in development, which will allow quasi-simultaneous magnetic field measurements of an FOV exceeding $50^{\prime \prime}$ in the photosphere and in the chromosphere. Another planned upgrade involves the $\mathrm{AO}$, which will be equipped with an $\mathrm{H}-\alpha$ filter and thus be able to lock on prominences and spicules near the solar limb, for instance, thus improving the image stabilization and quality for off-limb observations.

\section{Summary and conclusions}

GREGOR has undergone significant changes from 2018 to 2020. New relay optics allow us to reach diffraction-limited optical quality over the whole FOV. The new instrument distribution allows us to plan for future instrumentation upgrades. The replacement of the last Cesic mirror with one made of Zerodur improved the contrast noticeably. A new SJ system provides diffraction-limited context images in the photosphere and in the chromosphere. The AO was enhanced to optimize many of the previously manual telescope functions, such as tracking and focusing. The derotator functionality was enhanced and its orientation calibrated.

New alignment methods were developed and solutions were found to optimize the beam stability and reduce the vibrations. The telescope is now controlled by a dedicated GUI, which includes the functionality for all subsystems. The observing room was optimized, with dedicated work stations to control the telescope and instruments and a weather monitor to improve the safety of noticing weather-related telescope closure conditions. Most systems are documented with manuals, checklists, and technical notes, which are accessible in a version-controlled database, based on a model for documentation of space missions. Management and policy changes further improved the stability of the telescope and of consistently obtaining scientifically valuable data. 
Acknowledgements. Operating GREGOR would not be possible without the dedicated KIS technical staff who are all very gratefully acknowledged. In particular, we would like to thank Oliver Wiloth, Andreas Bernert, Stefan Semeraro, Frank Heidecke, Michael Weissschädel, Clemens Halbgewachs, Tobias Preis, Peter Markus, Peter Caligari, Marco Günter, Markus Knobloch, Roland Fellmann, Christine Fellmann, Bruno Femenia, Daniel Gisler, and Sylvia Nadler. We also thank Göran Scharmer and an anonymous referee for helpful comments on the manuscript. The 1.5-meter GREGOR solar telescope was built by a German consortium under the leadership of the Leibniz-Institute for Solar Physics (KIS) in Freiburg with the Leibniz Institute for Astrophysics Potsdam, the Institute for Astrophysics Göttingen, and the Max Planck Institute for Solar System Research in Göttingen as partners, and with contributions by the Instituto de Astrofisica de Canarias and the Astronomical Institute of the Academy of Sciences of the Czech Republic.

\section{References}

Berkefeld, T., Schmidt, D., Soltau, D., von der Lühe, O., \& Heidecke, F. 2012, Astron. Nachr., 333, 863

Berkefeld, T., Schmidt, D., Heidecke, F., \& Fischer, A. 2018, in Proc. SPIE, SPIE Conf. Ser., 10703, 107033A

Bianda, M., Berdyugina, S., Gisler, D., et al. 2018, A\&A, 614, A89

Collados, M., López, R., Páez, E., et al. 2012, Astron. Nachr., 333 872
Dhara, S. K., Capozzi, E., Gisler, D., et al. 2019, A\&A, 630, A67

Gandorfer, A. M., \& Povel, H. P. 1997, A\&A, 328, 381

Gisler, D., Berkefeld, T., \& Berdyugina, S. 2016, in Proc. SPIE, SPIE Conf. Ser., 9906, 99065E

Goode, P. R., Coulter, R., Gorceix, N., Yurchyshyn, V., \& Cao, W. 2010, Astron. Nachr., 331, 620

Hofmann, A., Arlt, K., Balthasar, H., et al. 2012, Astron. Nachr., 333, 854

Jing, J., Xu, Y., Cao, W., et al. 2016, Sci. Rep., 6, 24319

Kleint, L., \& Gandorfer, A. 2017, Space Sci. Rev., 210, 397

Penn, M. J. 2014, Liv. Rev. Sol. Phys., 11, 2

Puschmann, K. G., Denker, C., Kneer, F., et al. 2012, Astron. Nachr., 333, 880

Scharmer, G. B., Bjelksjo, K., Korhonen, T. K., Lindberg, B., \& Petterson, B. 2003, in Proc. SPIE, eds. S. L. Keil, \& S. V. Avakyan, SPIE Conf. Ser., 4853, 341

Scharmer, G. B., de la Cruz Rodriguez, J., Sütterlin, P., \& Henriques, V. M. J. 2013, A\&A, 553, A63

Scharmer, G. B., Löfdahl, M. G., Sliepen, G., \& de la Cruz Rodríguez, J. 2019, A\&A, 626, A55

Schmidt, W., von der Lühe, O., Volkmer, R., et al. 2012, Astron. Nachr., 333, 796

Schmidt, D., Gorceix, N., Marino, J., et al. 2016, in Proc. SPIE, SPIE Conf. Ser., 9909, 990929

von der Lühe, O., Schmidt, W., Soltau, D., et al. 2001, Astron. Nachr., 322, 353 von der Lühe, O., Volkmer, R., Kentischer, T. J., \& Geißler, R. 2012, Astron. Nachr., 333, 894 